\title{
Statistical Study of the Inter-Dot Excitation Transfer in CdTe/ZnTe Quantum Dots
}

\author{
M. Koperski ${ }^{a}$, T. KazimierczuK ${ }^{a}$, M. Goryca ${ }^{a}$, A. Golnik ${ }^{a}$, J.A. Gaj $^{a}$, M. NAwrocki $^{a}$, \\ P. WOJNAR ${ }^{b}$ AND P. KOSSACKI ${ }^{a}$ \\ ${ }^{a}$ Institute of Experimental Physics, Faculty of Physics, University of Warsaw, Hoża 69, 00-681 Warsaw, Poland \\ ${ }^{b}$ Institute of Physics, Polish Academy of Sciences, al. Lotników 32/46, 02-668 Warsaw, Poland
}

In this work we present a statistical study of resonantly excited luminescence of coupled $\mathrm{CdTe} / \mathrm{ZnTe}$ quantum dots studied by photoluminescence excitation measurements. We investigate the probability of resonance occurrence as a function of resonant energy. We come to the conclusion that the distribution of the inter-dot resonances is uniform, which suggests that the inter-dot excitation transfer is not limited by mean density of states in the emitting quantum dots.

PACS: 78.67.Hc, 78.55.Et

\section{Introduction}

The physics of $\mathrm{CdTe} / \mathrm{ZnTe}$ quantum dots (QDs) belongs to the popular field of research in semiconductor nanostructures. The quantum confinement of the carriers in a zero-dimensional system leads to the discrete density of states, which implies the existence of single photoluminescence (PL) lines in isolated QD spectrum. Those lines correspond to the radiative recombination of various charge complexes (e.g., excitons, trions) [1]. The carriers which form those complexes might be photocreated in the surrounding barriers by non-resonant optical excitation. They might be also created directly in a QD by absorption of the photons of energy corresponding to the excited state of the QD. In such a case the signal in photoluminescence excitation (PLE) measurements exhibits resonant behavior (strong increase for particular excitation energies). It has been shown that similar resonances occur also in the case of resonant excitation of spontaneously coupled QD pairs [2-4]. In such a scenario, the carriers are resonantly created in the absorbing QD. Then, they are transferred to the second QD, in which they recombine producing a photon (typical PLE spectra are shown in Fig. 1). The detailed mechanism of such a transfer still remains unclear, however, some possible explanations were proposed $[4,5]$. In this paper we present a statistical study of occurrences of resonances in PLE spectra for CdTe/ZnTe QDs.

\section{Samples and experimental setup}

The PLE measurements were performed on samples containing self-organized $\mathrm{CdTe} / \mathrm{ZnTe}$ QDs grown by the Stranski-Krastanov method. The QD formation was induced by tellurium desorption $[6,7]$. The density of
QDs estimated by atomic force microscopy was $10^{9}$ $10^{10} \mathrm{~cm}^{-2}$. In PLE measurements the samples were attached to a specially designed microscope objective and placed inside a cryostat. They were cooled down to $1.7 \mathrm{~K}$ with pumped helium. We used a tunable dye laser for excitation in the energy range 2020-2200 meV. The short-pass and long-pass filters were used to reject stray light. The microluminescence from the QDs was spectrally resolved by a spectrograph and measured with a CCD camera. For each PLE measurement we selected QDs from the low energy tail of the broad PL emission band. We selected spectrally isolated dots with typical $\mathrm{X}, \mathrm{X}^{+}, \mathrm{X}^{-}$, $\mathrm{XX}$ pattern as shown in Fig. 1. For each dot we performed PLE measurement in the range given by the dye laser.

\section{Statistical treatment of the resonances occurrence}

We have performed a detailed statistical analysis of over 700 occurrences of resonances (example in Fig. 1). We parameterized the position of the resonance with two values: the exciton energy in the QD exhibiting resonant luminescence and the exciton energy in the absorbing QD (i.e., the excitation energy for which the resonance was observed). The plot of correlation of these energies is presented in Fig. 2. We found no characteristic features in the distribution of resonances. Only small change in the density may be noticed. The highest density of the resonances was found in the area around the emission energy of $1940 \mathrm{meV}$, gradually decreasing in a range of about $100 \mathrm{meV}$.

The marginal distributions of the absorption and emission energies are presented in Fig. 3. The bars of the 


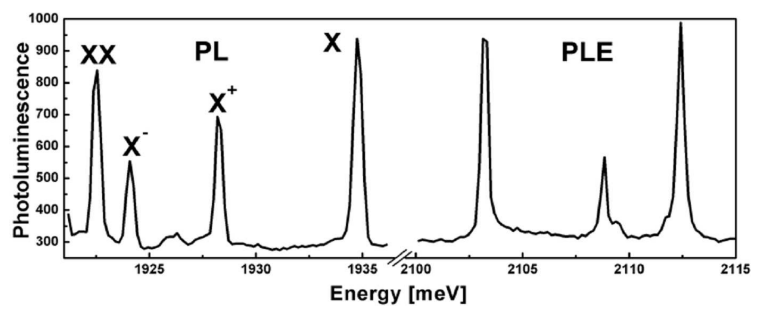

Fig. 1. An example of a resonant photoluminescence spectra of single quantum dot (left) and PLE monitored for the energy of exciton (right).

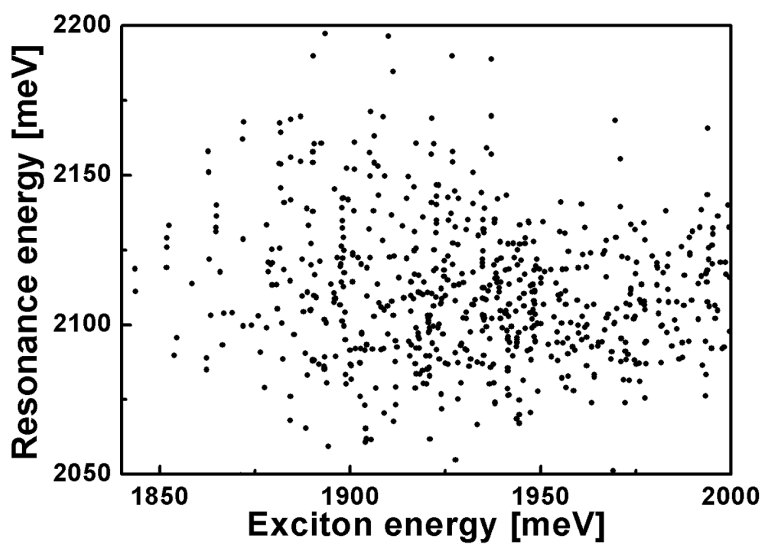

Fig. 2. The distribution of resonances depicted on the exciton energy-excitation energy plane.

left histogram represent the number of analyzed excitons for given energy. The bars of the right histogram show the probability of observing a resonance as a function of the excitation energy. This probability was estimated as a ratio between number of observed resonances in given energy range and the number of measurements performed for this range. These two distributions of exciton energy and excitation energy resemble the Gaussian function centered at the energies equal to 1920 and $2100 \mathrm{meV}$, respectively. A luminescence of the QD ensemble has been placed over the histograms for reference. We observe good correspondence between the maxima of the resonances distribution and the luminescence. It is important to note that while the right histogram represents the true probability of the observation of resonance, the left histogram is determined by our selection of emitting QDs from the tail of PL emission band. It is presented here for reference. Our main interest, however, is related to the distribution of probability of resonance occurrences as a function of excitation energy with respect to the exciton energy. The probability has been computed in the same manner as in the case of absolute energies.

The result in the form of a histogram is presented in Fig. 4. The obtained distribution is nearly uniform, although small maxima can be recognized. They might be associated to increased mean density of states in the

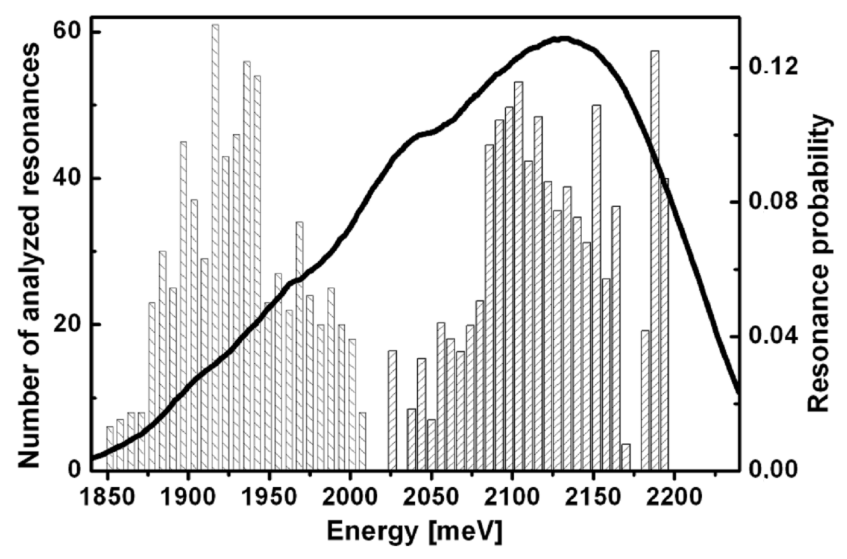

Fig. 3. The histogram of the exciton distribution for observed resonances (left histogram) and of the distribution of probabilities of resonance occurrences (right histogram). The solid line represents the QD ensemble photoluminescence.

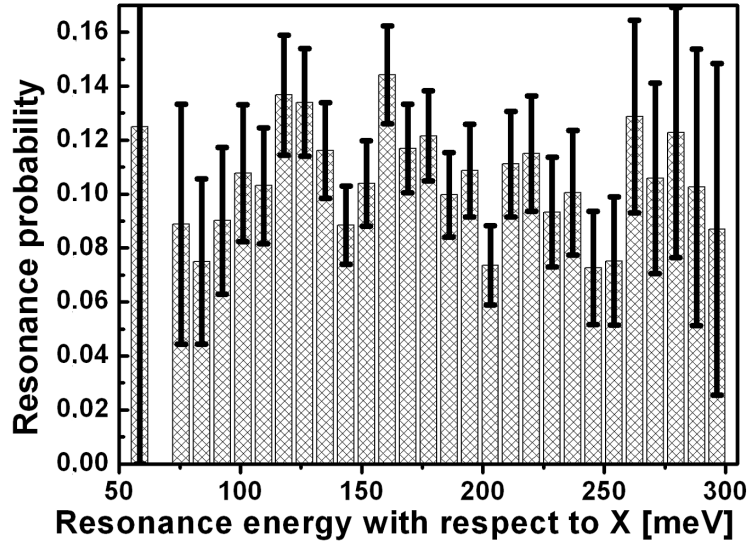

Fig. 4. The distribution of the probability of resonant luminescence as a function of resonance energy with respect to exciton energy.

region of energies for consecutive shells in the QD [8]. However, the amplitude of the questioned variations is small with respect to the statistical uncertainty even despite the large number of analyzed events. It is clear that the distribution does not follow any systematic increase similar to increase of the mean density of states $(\propto E)$ expected for 2D harmonic potential often assumed for self assembled QDs [9]. The uniform distribution can originate from very strong coupling between QDs. Then the mean density of states in the emitting dot does not limit the probability of occurrence of the efficient transfer. The transfer time is much shorter than the radiative time of the exciton [3] and the exact transfer rate does not affect the PL intensity. Moreover, in actual systems the discrete density of states in the QD becomes broadened due to short lifetime of the highly excited states. The energy position of higher excited shells also varies from dot to dot [10]. Taking all these things into account one 
may obtain the uniform distribution of resonance probabilities as a function of the energy of the resonance with respect to exciton energy.

\section{Conclusions}

We performed a statistical analysis of occurrence of coupled QDs pairs in a self-assembled CdTe/ZnTe QDs system. Results show that the occurrence of the efficient inter-dot transfer does not depend on the relative energies of the involved states (i.e. energy of the absorbing and emitting state). The shallow features observed in the resonance distribution might be associated with increased mean density of states at the energies corresponding to electronic shells in the QD.

\section{Acknowledgments}

This work was partially supported by the Polish Ministry of Science and Higher Education as research grants in years 2010-2014 (projects Iuventus Plus and NCBiR Lider).

\section{References}

[1] Y.Z. Hu, M. Lindberg, S.W. Koch, Phys. Rev. B 42, 1713 (1990).

[2] T. Kazimierczuk, S. Nowak, J. Suffczyński, P. Wojnar, A. Golnik, J.A. Gaj, P. Kossacki, Acta Phys. Pol. A 112, 321 (2007).

[3] T. Kazimierczuk, J. Suffczyński, A. Golnik, J.A. Gaj, P. Kossacki, P. Wojnar, Phys. Rev. B 79, 1533011 (2009).

[4] M. Goryca, T. Kazimierczuk, M. Nawrocki, A. Golnik, J.A. Gaj, P. Kossacki, P. Wojnar, G. Karczewski, Phys. Rev. Lett. 103, 087401 (2009).

[5] T. Kazimierczuk, A. Golnik, P. Wojnar, J.A. Gaj, P. Kossacki, Phys. Status Solidi B 247, 1409 (2010).

[6] F. Tinjod, B. Gilles, S. Moehl, K. Kheng, H. Mariette, Appl. Phys. Lett. 82, 4340 (2003).

[7] P. Wojnar, J. Suffczyński, K. Kowalik, A. Golnik, M. Aleszkiewicz, G. Karczewski, J. Kossut, Nanotechnology 19, 235403 (2008).

[8] M. Fricke, A. Lorke, J.P. Kotthaus, G. Medeiros-Ribeiro, P.M. Petroff, Europhys. Lett. 36, 197 (1996).

[9] M. Vachon, S. Raymond, A. Babinski, J. Lapointe, Z. Wasilewski, M. Potemski, Phys. Rev. B 79, 165427 (2009).

[10] K. Kukliński, Ł. Kłopotowski, K. Fronc, M. Wiater, P. Wojnar, P. Rutkowski, V. Voliotis, R. Grousson, G. Karczewski, J. Kossut, T. Wojtowicz, Appl. Phys. Lett. 99, 141906 (2011). 